Delft University of Technology

\title{
Quantifying automatable checklist items on a commercial flightdeck
}

Reitsma, J.P.; van Paassen, M.M.; Borst, C.; Mulder, Max

DOI

10.2514/6.2021-1319

Publication date

2021

Document Version

Final published version

Published in

AIAA Scitech 2021 Forum

\section{Citation (APA)}

Reitsma, J. P., van Paassen, M. M., Borst, C., \& Mulder, M. (2021). Quantifying automatable checklist items on a commercial flightdeck. In AIAA Scitech 2021 Forum: 11-15 \& 19-21 January 2021, Virtual Event [AIAA 2021-1319] American Institute of Aeronautics and Astronautics Inc. (AIAA). https://doi.org/10.2514/6.20211319

\section{Important note}

To cite this publication, please use the final published version (if applicable).

Please check the document version above.

\section{Copyright}

Other than for strictly personal use, it is not permitted to download, forward or distribute the text or part of it, without the consent of the author(s) and/or copyright holder(s), unless the work is under an open content license such as Creative Commons.

Takedown policy

Please contact us and provide details if you believe this document breaches copyrights.

We will remove access to the work immediately and investigate your claim. 


\title{
Quantifying automatable checklist items on a commercial flightdeck
}

\author{
Jelmer P. Reitsma, M.M. (René) van Paassen, Clark Borst, and Max Mulder* \\ Delft University of Technology, Delft, 2629HS, The Netherlands
}

\begin{abstract}
In-flight non-normal events can be rather taxing for a flight crew. Numerous tasks, often competing for attention, need to be handled adequately after which, the best plan of action for the remainder of the flight needs to be determined. In the light of recent developments towards reduced crew operations, the demand for reducing workload has become apparent. This requires us to rethink the role of the pilot, which to the authors perspective is mainly one of a flying and flight plan manager. System management is a function that can be assigned to automation. Automation on modern plane often already monitors systems more accurate and faster than pilots can every do. However, in this study we explore the potential checklist step reduction if, the automation will go one step further. Namely, automatically execute reconfiguration steps that do not affect flight characteristic. In total, $39 \%$ of the checklist items are potential candidates for this new automation. Average checklist size can be reduced to 4 , compared to the current average of 6.5 items per checklists. This result does not provide us with an estimated time saving. Although, the result seems promising to reduce the workload on the flight crew. This exploration can be followed-up by a study to estimate the potential time savings. Besides this improvements can be made to reduce the length of the informative statements and implications on the flight plan can be presented in a more efficient manner.
\end{abstract}

\section{Introduction}

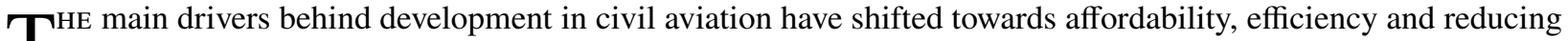
the environmental impact. Where fuel efficiency gains become more difficult to achieve, costs savings through Reduced Crew Operation (RCO) seem worth exploring. These new concepts come with mainly human factor related challenges that need to be resolved in order to be viable for real world application. By removing a crew member from the flight deck, the already existing high workload peaks, e.g., during non-normal events, are enlarged and may become unmanageable. Studies on reduced crew operations within the current flight deck [1] found that the checklist completion time during system malfunctions significantly increases during single pilot operations compared to the conventional two crew set-up, to 20 minutes compared to 3 minutes, respectively. Future flight safety might be jeopardized, if we do not critically rethink the flight-deck and the role of the pilot.

However, not only on single pilot operation concepts can high workload situations be an problem. Also, on current modern flight decks completing the correct checklists can be a time consuming process. Take for example the Qantas Flight 32 incident where a five person crew took over 50 minutes to complete all of the initial procedures associated with the alert messages after an un-contained engine failure [2].

It seems that crews, regardless the size, could benefit from automation that completes the associated procedures by re-configuring systems as appropriate [1] [3]. This will have the potential to lower pilot workload, during non-normal events, and allows the crew to focus on what is really important, i.e., flying the plane and plan the remainder of the flight. Planning the remainder of the flight can be complex. Accident and incident reports show that crews have difficulty to extract and remember the implications that a particular malfunction might have for their continued flight [4][5]. Burian [4] describes that crews are benefiting from short checklists during abnormal events. So, if we would relief the pilot from all or a majority of the reconfiguration steps in the procedures the likelihood that pilots can devote sufficient attention to planning and executing a contingency plan increases.

Not only will automatic system reconfiguration likely reduce workload. Increased automation has also the potential to resolve issues regrading incorrect execution of the checklist. Where the electronic checklist helped to solve checklist errors like skipping a checklist or omitting a checklist line item [6], a fully automated re-configuring system may also eliminate incorrect switching actions, e.g., selecting the bleed switch instead of a pack switch [7].

*Control \& Simulation, Department Control \& Operations, Delft University of Technology, Delft 
No doubts exist if the technology is ready to do automatic re-configuration of the system, since many system management tasks are already done automatically. Take for example, on the Boeing 737-800, automatic load shedding in case of electrical malfunctions and automatic re-configuring of pack inflow during an air conditioning pack malfunction. However, some items cannot be practically and reliably sensed or performed by automation. Take for example closing doors, establish crew communications or looking out the window to check the wing. These items remain something that the human needs to do. But many of the items that are already controlled or sensed through systems seem perfect candidates to automate.

Automation on legacy planes was added through an evolutionary, technology-driven process. This resulted into a patchwork of automated systems which is far from ideal. A holistic approach towards automation is beneficial for clarity and simplicity for the operators. Therefore, this proposed automatic re-configuration system is targeted at future flight-decks where the holistic view approach can be applied. But it would also be possible to retrofit all controls and selectors, maybe even only digitally. The most important feature of the retrofitted flight-deck is that selector positions need to correspond with what the automation did, so no confusion can occur.

Within literature many authors suggest that systems should become increasingly automated to lower workload [1][3]. But to date, no literature was found that provides a quantification of what the potential benefits could be. In other words, how much shorter can the checklists become if we would automate the system management tasks? As a starting point, we apply this analysis to a Boeing 737-800 (Boeing 737-86D) since this would be an plane suitable for single pilot operations due to the type of missions these planes fly. This study will also provide a break-down of the structure of a common Quick Reference Handbook (QRH) that can provide insight into the current available guidance material on the flight-deck. Deviations due to additionally equipped systems are considered small and not altering the result too much compared to other 737 .

\section{Method}

As mentioned earlier, the question is no longer if system reconfiguration steps can be automated but rather if they should be automated. Possibilities seem endless, but from the past, we learned how the introduction of automation can lead to skill degradation and out-of-the-loop events. Designers should carefully reconsider what to automate and how to do this appropriately without introducing potential problems. Since the QRH contains basically steps of all tasks we need to determine what we want to automate and especially what not.

\section{A. Assumed pilot's role}

As we have learn from the past, humans are poor monitors and not good in routine tasks, nor memorizing. But humans can be creative and can act on their intuition. On the other hand, automation is not good at dealing with events for which it is not programmed. Today the pilot is a flyer, flight path controller, systems monitor and flight deck manager. In this paper, we envision a shift in the role of the pilot. The pilot would be more involved and focused on flying, flight path management and route planning. Planning requires often a knowledge base, intuition and creativity, something that is difficult to capture in a system. Finding a suitable path and environment for the jeopardized systems is something that the human is good at since it requires intuition and creativity. The pilot is released from the task of troubleshooting, i.e., diagnosing, monitoring, and re-configuring the system, which are all routine and well defined tasks.

Systems on modern planes have become very complex. Understanding and diagnosing the entire system to a deep technical level is almost impossible. Especially, under high workload and stressful situations. The system has better accessibility to relevant system information and is also more accurate in interpreting this data. Furthermore, once a limit has been exceeded the pilot is expected to follow the prescribed procedure, almost like a machine. This eliminates the pilot strengths of being creative or intuitive. The question is why do we ask the pilot to do this task in the first place? On modern planes many systems have redundant components. Malfunctions can often be contained by switching on the redundant system and flight safety is rarely endangered. However, the contained but inoperative systems can impose limitations to the remainder of the flight. Which can have grave consequences if the pilot is unaware. Hence, these items can not be left out.

\section{B. Automation candidates}

In-line with the previously discussed pilot's role as a flyer, some items that can technically be automated (and sometimes already are) are deliberately chosen not to be automated. These items include actions that are impacting flight characteristics significantly. Why? Well, this has all to do with keeping the pilot engaged. Pilots fulfill a role 
as a back-up. Once the autopilot cannot cope with the situation, the pilot has to take over. Being aware of the basic configuration the plane is crucial to keep the pilot in-the-loop. These basic configuration items include thrust settings, gear levers, flaps stetting, speed-brakes and trim settings. These items are not suitable to be automated.

Reconfiguration steps that handle resource systems, e.g., hydraulics, electrical, fuel and pneumatic systems are good candidates to be handled automatically. This is because these systems are often redundant, the system are already monitored by the plane, clear limitations exist and the procedures are also clearly defined.

Plane and passenger health protection systems are also suitable candidates. Often these tasks are time critical, the corrective action is well defined, and the systems are also equipped with sensors. Furthermore, options for resolution are often limited.

To generalize, the described tasks above are tasks that have the main objective to (1) protect the plane and passengers from harm, or (2) provide comfort, or (3) maximize the performance of the systems on-board the plane. If checklist items serve on or more of these goals, then they are often good candidates for automation.

If we look at Figure 1a, we can see that many of the steps belong to this category. Checklist item 1, 2, 3, and 4 are all candidates to be automated. This is also true in another example, shown in Figure $1 \mathrm{~b}$ Items 1, 2, 3 and 4 can be automated. However, item 4 requires some context. Ice can be expected below 40,000ft, in clouds and if the outside air temperature is below or near the freezing point. To determine this, some integration of the systems is required. Furthermore, it can be linked to SIGMETs that report or predict icing conditions.

In Figures $1 \mathrm{a}$ and $1 \mathrm{~b}$, we see clear actions. These are presented with the selector on the left side, dots in the middle and a target state on the right. The example checklists show also choose items decision statements. In this case, the pilot has to decide what condition applies. These items are often already auto-sensed, on planes that are equipped with electric checklists. They are relatively easy to automate since these options often describe if a light is illuminated or extinguished, which are already sensed by the system.

Conditional statements along with objective statements presented on top of the checklist, in a grey box (see Figure 1a and 1a, are no actions and therefore out of the consideration to be automated. This also holds for operational notes and informative statements, describing the expected behavior of the systems after the action is performed.

To summarize, the items that are selected to be suitable candidates for automation in this study are as follows:

- Actions that have the primary function to protect the plane and passengers from harm, or maximize the functionality of the plane. And that do not directly impact flight characteristics.

- Decision statements that can be measured with sensors.

With these criteria, the each item in the QRH is being rated if they would be suitable candidates for automation. Firstly, all items in the QRH are categorized into actions, notes or remarks, decision statement, and conditional statements, which describe for what situation the checklist can be used. These items are sequentially categorized into 'automatic', 'manual', 'informative', 'wait-until' or 'go-to-next-checklist' items. Wait items are, as the name suggests, items that describe to wait a certain duration or until a certain event occurred. An example of such items are 'wait for 2 minutes' or 'wait until the light has extinguished'. Some actions are not necessarily straight forward actions, sometimes they are more of an informative nature for operational purposes, for example, 'continue normal operations'. Therefore these are marked as informative statements, providing information for the remainder of the flight.

\section{Results}

In total 159 checklists are analyzed, both normal and non-normal including the (short) deferred checklists and items. All these checklists contain to a total of 1626 items. Of all the 1626 items, $68 \%$ is classified as an action, $13 \%$ as a condition that needs to be verified (choose item or decision statement), $10 \%$ as a conditional statement (items presented on top of the checklist) and finally $9 \%$ of the items are notes or remarks. This result is presented in Table 1

From Table 1 , we can obtain that $36 \%$ of all items can be automated. The wait items are also potential candidates for automation since they require only a timer. Items that state to go to a follow-up checklists are also good candidates to be automated. By adding up all these items, we can find that a total of $39 \%$ of the items, currently in the QRH, can be automated. Leaving the pilot to read and execute $61 \%$ of items presented in the QRH.

Some notes and remarks are presented just below an action to provide some information about the expected system behavior. These items can become redundant if the automation is handling these items. This means that the number of informative items for the proposed automated system will be reduced as well. However, a critical assessment is required to determine if these items are superfluous. This information is often added, it seems, to prevent surprises, distinguishing normal from normal system behavior and increase trust in the system. 


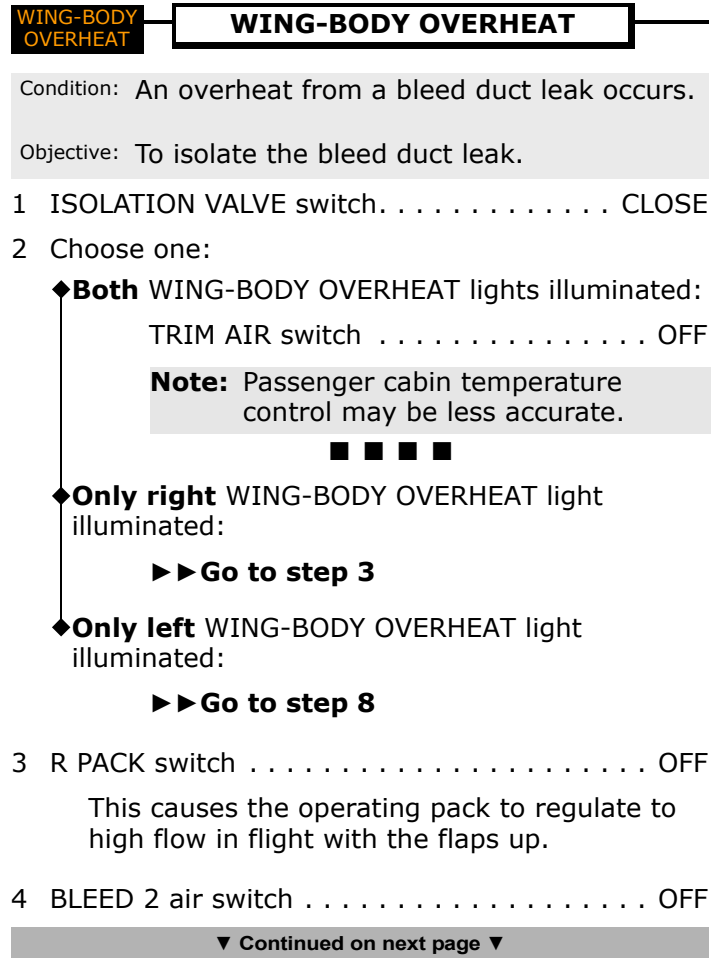

(a) Checklist containing actions, decision items, notes and remarks on the to-be-expected system behavior.

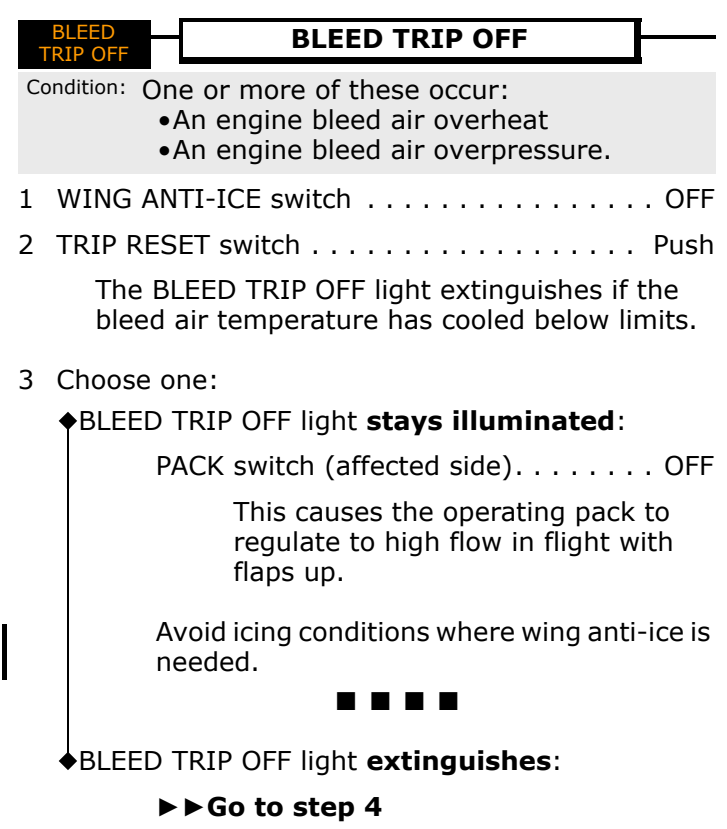

4 WING ANTI-ICE switch . . . . . . . . As needed

Caution! Use of wing anti-ice above
approximately FL350 may cause bleed
trip off and possible loss of cabin
pressure.

$\square \mathbf{a}$

(b) Another checklist containing actions, which have context specific target states and remarks that are important for the remainder of the flight.

Fig. 1 Example checklists extracted for a Quick Reference Manual (QRH) of a Boeing 737-800, as presented in [8].

Table 1 Checklist item step break down of 10 normal checklist, 12 deferred checklist/procedures and 137 non-normal checklists showing the potential of

\begin{tabular}{rccccc}
\hline Step Classification & Action Item & Decision item & Conditional statement & Notes \& Remark & Total \\
\hline Automatable items & 411 & 175 & - & - & $586(36 \%)$ \\
Informative items & 244 & - & 164 & 138 & $546(34 \%)$ \\
Manual items & 405 & 34 & - & 5 & $444(27 \%)$ \\
Go to next checklist item & 40 & - & - & - & $40(2 \%)$ \\
Wait items & 10 & - & $164(10 \%)$ & $143(9 \%)$ & $1626(100 \%)$ \\
\hline Total & $1110(68 \%)$ & $209(13 \%)$ & & &
\end{tabular}

In Figure 2, the amount of occurrences of the same number of items per checklists are presented. What is interesting, but not surprising, is that for the automated checklists, the number of checklist items is reduced. The extreme long checklists with $>30$ items are mitigated. The average checklist size is reduced to 4 for the automated checklist, compared to 6.5 items for the current system.

For the automatic checklist, the remaining items are for $55 \%$ informative and $45 \%$ manual action items. The checklist includes mainly items directly related to flying, flight plan management, or communication. Therefore, the 


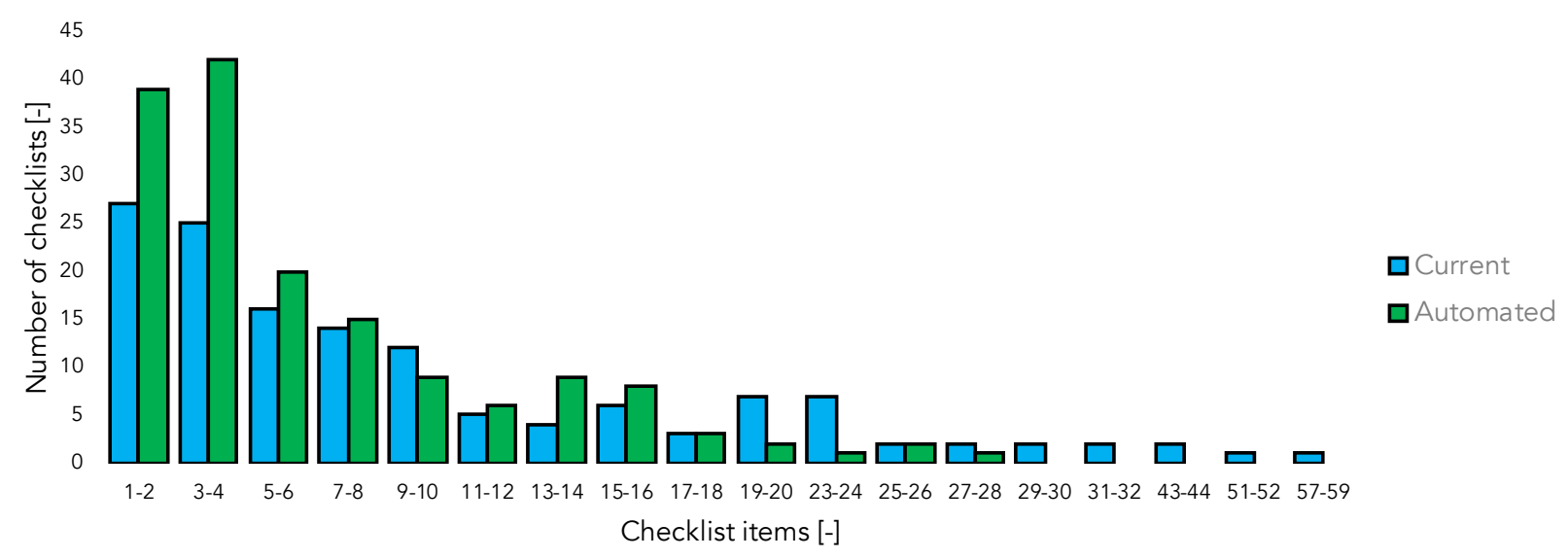

Fig. 2 Occurrences of the number of items per checklist. Note the shift towards the left for the automated checklist concept.

action items can be compared to instructions a flight instructor would give.

\section{Discussion}

The question remains if this result is sufficient to proceed with the development of such automation. This decision depends mainly on investments of modification, maintainability or development of these systems and expected cost savings, of for example RCO, or expected increase of level of safety.

\section{A. Modifications to the plane}

The automation would require major adjusts of the systems. The system would require integration of sensed data, and a structured way of dealing with data. All this is not technologically un-imaginable. The most modern airplane architectures have a internal network that allows for data access across the plane. This new automation tap on to this network and handle appropriately. However, it would be easier to implement this on newly designed planes rather than retrofitting planes.

\section{B. Need for information integration}

One of the challenges is to provide context from outside the plane into this automated assistant. But again this is not impossible, since modern day cars are already equipped with, for example, speed limit indication based on their current location. This would also be possible in aviation, were information is managed in a more structured.

\section{Controls and switches}

Although items are being automated, the pilot, we think, should still be able to control the items in cases of unexpected events. The question however is, do these controls and switches need to be as present as they are today. Since they are not used as much as before, maybe sporadically, they could be presented digitally on displays and/or integrated in a synoptic view, leveraging on touchscreen technology.

\section{Saving items does not correlate to time saving}

Although the results show that checklists can be shortened by letting automation perform many of the items, it doesn't directly translate into similar results of time saved. To estimate the time saved by the automation we need to measure the time how long each item takes to be execute. This can either be done physically or estimated with for example the well known Fitts Law. Furthermore, items with much text, like operational notes, take much more time than shorter action text items. The time to read these items can be estimated with for example an average reading speed ranging form 175 to 300 words per minute [9]. This analysis is quite labor intensive.

Also the checklists are structure as troubleshooting trees. Based on the decision along throughout the process, the checklist will be longer or shorter. In order to investigate this properly one has to determine all possible combinations of 
the decisions made while troubleshooting. This will provide a more accurate number for real life operations.

Although the analysis could be enhanced with the above-mentioned, this study served its purpose. This study was intended to obtain an initial quantification of the potential canditates for automation on board commercial aircraft, which seem promising. Next would be to determine all possible combinations and determine the time spend per item and checklist.

\section{E. Legacy plane}

The Boeing 737 was introduced in 1968, since then it went through many modifications and adjustments. This plane has some automation, but does not incorporate the level of integration the most modern jets have. The QRH of the Boeing 777 and 787 is more compact, more structured. The ECL automated already many items, i.e., the decision items. Therefore, the benefits of implementing more automation on these planes is less compared to a less integrated plane like the Boeing 737. On the other hand, modifying these planes will be less drastic and therefore more economical.

\section{F. Actions sometimes not really actions}

Lastly, it was found that the structure of the QRH is sometimes not clear. Some items are presented as actions, while the are more of an informative nature. It seems not clear when an item is presented as a note or as an action. This is occurs less on the new planes.

\section{Conclusion}

This exploratory study provides promising results in the journey to develop a flight-deck with lower workload. Almost $39 \%$ of all checklist items are possible candidates to be automated. This result was obtain with the assumption that the pilot's role will be more focused flying and flight plan management. Checklist items directly related to flying are deliberately not automated to keep the pilot in the loop. The average checklist size is decreased from 6.5 to 4 for the baseline and automated concept, respectively. This does not entail that $39 \%$ of the time is saved. Although, the reduction in terms of checklist items is significant. Planes require integration of information to make this automation possible. Even the most modern planes, that are equipped with electronic checklist which can sense the state of the system, can benefit from this concept. This is because the automation will not only perform decision items automatically but also executes them, and taking the automation to the next level. In all cases it will likely reduce workload, the question however to what costs?

\section{References}

[1] Bailey, R. E., Kramer, L. J., Kennedy, K. D., Stephens, C. L., and Etherington, T. J., “An Assessment of Reduced Crew and Single Pilot Operations in Commercial Transport Aircraft Operations," ????

[2] Australian Transport Safety Bureau, "In-flight uncontained engine failure A380-842, VH-OQA," ATSB Transport Safety Report, , No. June, 2013, pp. 1-305.

[3] Harris, D., "A human-centred design agenda for the development of single crew operated commercial aircraft," ???? https://doi.org/10.1108/00022660710780650

[4] Burian, B. K., Barshi, I., and Dismukes, R. K., “The Challenge of Aviation Emergency and Abnormal Situations,” NASA Technical Memorandum, , No. June, 2005, pp. 1-21.

[5] Reitsma, J., Fucke, L., Borst, C., and van Paassen, R., "Taking a Closer Look at Flight Crew Handling of Complex Failures - Ten Case Studies," 19th International Symposium on Aviation Psychology (ISAP 2017), Dayton, 2017.

[6] Boorman, D., "Safety benefits of electronic checklists: An analysis of commercial transport accidents," Proceedings of the 11th International Symposium on Aviation Psychology, Columbus, 2001, p. 5-8. URL http://www.faa.gov/library/online_libraries/ aerospace_medicine/sd/media/Boorman.pdf

[7] AAIB, “Air Accident Monthly Bulletin February 2011,” Tech. rep., AAIB, 22011.

[8] Boeing, "Boeing 737-800 Quick Reference Handbook," 2007.

[9] Brysbaert, M., "How many words do we read per minute? A review and meta-analysis of reading rate," , 042019. https://doi.org/10.31234/osf.io/xynwg 